\title{
Two standard flora of the lower reaches of the Lena River, as an indicator of the territory's biodiversity
}

\author{
Evgenii Nikolin ${ }^{1,2 *}$, and Irina Yakshina ${ }^{2}$ \\ ${ }^{1}$ Institute for Biological Problems of Cryolithozone SB RAS, 677980 Yakutsk, Russia \\ ${ }^{2}$ State Nature Reserve Ust-Lensky, 678400 Tiksi, Republic of Sakha (Yakutia), Russia
}

\begin{abstract}
The standard flora method established the diversity of vascular plants in two areas of the lower reaches of the Lena River (North-East Asia), which is estimated at 232 (216 species, 1 notospecies, 11 subspecies and 4 varieties) - 241 (228 species, 11 subspecies and 2 varieties) taxon $/ \mathrm{km}^{2}$.
\end{abstract}

The problem of obtaining comparable quantitative data on the diversity of vascular plants per unit area has already been covered by us in the collection BIO Web of Conferences [1]. In this case, information is provided about two new sites studied in the volume of standard flora. Both sites are located in the right-bank part of the lower reaches of the Lena River, about $20 \mathrm{~km}$ downstream from the island of Tit-Ary, within the northern spurs of the Kharaulakh ridge. The landscape of the studied area is mountainous-tundra, with altitudes up to $300 \mathrm{~m}$. Among the predominant tundra vegetation, there are small fragments of tree and shrub communities. According to the standard flora method, the area of each plot is 1 $\mathrm{km} 2$. One of the sections (SF 1) covers the estuary part of the right tributary of the Lena River - the Chinke River, the other (SF 2) - the estuary part of the Lena tributary similar in volume to the catchment area - the Sobol-Yuryage River. Since the distance between the mouths of these rivers is small, about $1 \mathrm{~km}$, the northwest corner of SF 1 and the southwest corner of SF 2 are combined at the same coordinate point.

The angular boundaries of the standard floras were highlighted in the coordinates: SF 1: extreme north-western point - 72 $07^{\prime} 27.47^{\prime \prime} \mathrm{N}, 126^{\circ} 58^{\prime} 43.95^{\prime \prime} \mathrm{E}$ - the bank of the Lena River below the mouth of the Chinke River; extreme north-eastern point $-72^{\circ} 07^{\prime} 27.98^{\prime \prime} \mathrm{N}$, $127^{\circ} 00^{\prime} 31.03 " \mathrm{E}$ - western exposure mountain slope in the Chinke and Sobol-Yuryage interfluve; extreme south-western point - 72 $06^{\circ} 55.24^{\prime \prime} \mathrm{N}, 126^{\circ} 58^{\prime} 46.29^{\prime \prime} \mathrm{E}-$ the bank of the Lena River above the mouth of the Chinke River; extreme south-eastern point $72^{\circ} 06{ }^{\prime} 55.60^{\prime \prime} \mathrm{N}, 127^{\circ} 00^{\prime} 28.91 " \mathrm{E}$ - mountain slope in the area between the Chinka and Lena rivers.

SF 2: extreme southwestern point $-72^{\circ} 07^{\prime} 27.47^{\prime \prime} \mathrm{N}, 126^{\circ} 58^{\prime} 43.95^{\prime \prime} \mathrm{E}$ - the bank of the Lena River in the Chinke and Sobol-Yuryage interfluve; extreme south-eastern point $72^{\circ} 07^{\prime} 34.92^{\prime \prime} \mathrm{N}, 127^{\circ} 00^{\prime} 26.14^{\prime \prime} \mathrm{E}$ - western exposure mountain slope in the Chinke and Sobol-Yuryage interfluve; extreme north-western point - $72^{\circ} 08^{\prime} 00.37^{\prime \prime} \mathrm{N}, 126^{\circ} 58^{\prime} 19.98^{\prime \prime} \mathrm{E}$

\footnotetext{
* Corresponding author: enikolin@yandex.ru
} 
- the bank of the Lena River below the mouth of the Sobol-Yuryage river; extreme northeastern point $-72^{\circ} 08^{\prime} 07.26^{\prime \prime} \mathrm{N}, 127^{\circ} 00^{\prime} 04.79^{\prime \prime} \mathrm{E}$ - the slope of the mountain in the interfluve of Sobol-Yuryage and Lena.

\section{List of species marked in the standard flora of Chinke and Sobol-Yuryage}

(numbers in parentheses indicate the corresponding numbers of the standard flora)

Fam. Cystopteridaceae (Payer) Schmakov: Cystopteris dickieana R. Sims (2); C. fragilis (L.) Bernh. (2).

Fam. Woodsiaceae (Diels) Herter: Woodsia glabella R. Br. (2).

Fam. Equisetaceae Rich.: Equisetum arvense L. (1, 2); E. palustre L. (1, 2); E. scirpoides (1, 2); E. variegatum Schleich. ex Web. et Mohr (1, 2).

Fam. Huperziaceae Rothm.: Huperzia arctica (Tolm.) Sipliv. (2).

Fam. Pinaceae Spreng. ex Rudolphi: Larix dahurica Turcz. subsp. cajanderi (Mayr) Dyl. (1).

Fam. Poaceae Barnh.: Alopecurus alpinus Smith. (1, 2); Arctagrostis arundinacea (Trin.) Beal. (1); A. latifolia (R. Br.) Griseb. (1, 2); Arctophila fulva (Trin.) Anderss. (1, 2); Bromopsis sibirica (Drob.) Peschkova (1, 2); Calamagrostis holmii Lange (1, 2); C. langsdorffii (Link) Trin. (1, 2); C. lapponica (Wahlenb.) C. Hartm. (1); C. neglecta (Ehrh.) Gaertn., Mey. et Schreb.: a). subsp. groenlandica (Schrank) Matuszk. (1, 2); б). subsp. neglecta (1, 2); Deschampsia borealis (Trautv.) Roshev. (1, 2): D. glauca C. Hartm. (2); D. obensis Roshev. (1, 2); D. submutica (Trautv.) Nikiforova (1, 2); D. sukatschewii (Popl.) Roshev.; D. $\times$ vodopjanoviae Nikiforova $(1,2)$; Dupontia fisheri subsp. pelligera (Rupr.) Tzvelev (1, 2); D. psilosantha Rupr. (1, 2); Elymus subfibrosus (Tzvelev) Tzvelev (1, 2); Festuca auriculata Drob. (1, 2); F. edlundiae S. G. Aiken (1); F. rubra L.: a). subsp. arctica (Hackel) Govor. (1, 2); б). subsp. rubra (1, 2); Hierochloe alpina (Sw.) Roem. et Schult. (1, 2); H. pauciflora R. Br. (1); Hyalopoa lanatiflora (Roshev.) Tzvel. (1); Koeleria asiatica Domin (1, 2); Leymus interior (Hult.) Tzvel. (1, 2); Poa alpigena (Blytt) Lindm.: a). subsp. alpigena $(1,2)$; б). subsp. colpodea (Th. Fries) Jurtzev et Petrovsky (1, 2); P. arctica R. Br. $(1,2)$; P. attenuata Trin. (2); P. bryophila Trin. (1, 2); P. filiculmis Roshev. (1); P. glauca Vahl (1, 2); P. pratensis L. s.str. (1); Trisetum molle Kunth (1); T. sibiricum Rupr. subsp. litorale Rupr. ex Roshev. (1, 2); T. spicatum (L.) K. Richt. (1, 2).

Fam. Cyperaceae Juss.: Carex aquatilis Wahlenb.: a). subsp. aquatilis $(1,2)$; б). subsp. stans (Drej.) Hult. $(1,2)$; C. bigelowii Torr. ex Schwein.: a). subsp. arctisibirica (Jurtz.) A. et D. Love $(1,2)$; б). subsp. ensifolia (Turcz. ex Gorodk.) Holub (1, 2); в). subsp. rigidioides (Gorodk.) Egor. (1); C. capillaris L. (1, 2); C. fuscidula V. Krecz. ex Egor (1); C. ledebouriana C. A. Mey. ex Trev. (2); C. melanocarpa Cham. ex Trautv. (1); C. rariflora (Wahlenb.) Smith. (1); C. rupestris All. (1, 2); C. vaginata Tausch. subsp. quasivaginata (C. B. Clarke) Malyschev (1); Eriophorum angustifolium Honck. (1, 2); E. scheuchzeri Hoppe $(1,2)$; E. tolmatchevii Novoselova (2); E. vaginatum L. (1, 2); Kobresia filifolia (Turcz.) Clarke (1, 2); K. myosuroides (Vill.) Fiori (1, 2); K. sibirica (Turcz. ex Ledeb.) Boeck. (1, 2); K. simpliciuscula (Wahlenb.) Mackenz. subsp. subholarctica Egor. (1).

Fam. Juncaceae Juss.: Juncus biglumis L. (1, 2); J. castaneus Smith (2); Luzula confusa Lindeb. (1, 2); L. multiflora (Ehrh. et Retz.) Lej. s.1. (1, 2); L. nivalis (Laest.) Spreng. (1, 2); L. tundricola Gorodk. ex V. Vassil. (1, 2).

Fam. Melanthiaceae Batsch ex Borch.: Tofieldia coccinea Richards. (1, 2); Veratrum oxysepalum Turcz. (1, 2); Zigadenus sibiricus (L.) A. Gray (2).

Fam. Liliaceae Juss.: Lloydia serotina (L.) Reichenb. $(1,2)$.

Fam. Orchidaceae Juss.: Coeloglossum viride (L.) C. Hartm. (1, 2). 
Fam. Salicaceae Mirb.: Salix abscondita Laksch. (1); S. alaxensis Coville $(1,2)$; S. arctica Pall. (1, 2); S. berberifolia Pall.: a). subsp. berberifolia (2); б). subsp. fimbriata A. Skvorts. (2); в). subsp. tschuktschorum (A. Skvorts.) Kuv. (2); S. boganidensis Trautv. (2); S. dasyclados Wimm. (1); S. fuscescens Andersson (1, 2); S. glauca L. (1, 2); S. hastata L. $(1,2)$; S. lanata L. $(1,2)$; S. polaris Wahlenb. $(1,2)$; S. pulchra Cham. $(1,2)$; S. recurvigemmis A. K. Skvortsov (1, 2); S. reptans Rurp. (1, 2); S. reticulata L. (1, 2); S. saxatilis Turcz. ex Ledeb. (1, 2); S. sphenophylla A. K. Skvortsov $(1,2)$; S. udensis Trautv. et C. A. Mey. (2).

Fam. Betulaceae S. F. Gray: Betula divaricata Ledeb. (2); B. nana L. subsp. exilis (Sukacz.) Hult. (1, 2); Duschekia fruticosa (Rupr.) Pouzar (1, 2).

Fam. Polygonaceae Juss.: Acetosa lapponica (Hiit.) Holub (1, 2); A. pseudooxyria (Tolm.) Tzvel. (1, 2); A. thyrsiflora (Fingerh.) A. L $\square$ ve et D. L $\square$ ve (1); Aconogonon ocreatum (L.) Hara (1, 2); A. tripterocarpum (A. Gray) Hara (1, 2); Bistorta plumosa (Small) D. Love $(1,2)$; B. vivipara (L.) Delabre (1, 2); Oxyria digyna (L.) Hill $(1,2)$; Rumex arcticus Trautv. (1); R. sibiricus Hult. (1).

Fam. Portulacaceae Juss.: Claytonia arctica Adams (1, 2).

Fam. Caryophyllaceae Juss.: Cerastium beeringianum Cham. et Schltdl:: a). subsp. beeringianum (2); б). subsp. bialynickii (Tolm.) Tolm.; C. jenisejense Hult. (1, 2); Dianthus repens Willd. (1, 2); Eremogone formosa (Fisch. ex Ser.) Fenzl (1, 2); Gastrolychnis affinis (Vahl ex Fries) Tolm. et Kozhan. (1, 2); G. uniflora (Ledeb.) Tzvel. (1, 2); Minuartia arctica (Stev. ex Ser.) Graebn. (1, 2); M. macrocarpa (Pursh) Ostenf. (1, 2); M. rubella (Wahlenb.) Hiern; M. verna (L.) Hiern (1, 2); Sagina nodosa (L.) Fenzl (1, 2); Silene chamarensis Turcz. subsp. paucifolia (Ledeb.) Kuvaev $(1,2)$; Stellaria ciliatosepala Trautv. $(1,2)$; S. crassifolia Ehrh. (1, 2); S. dahurica Willd. ex D. F. K. Schltdl. (1); S. edwardsii R. Br. ex Rich. (1, 2); S. longifolia Muehl. ex Willd. (1); S. palustris Retz. (1); S. peduncularis Bunge. (1); Wilhelmsia physodes (Fisch. ex Ser.) McNeill $(1,2)$.

Fam. Ranunculaceae Juss.: Anemonidium richardsonii (Hook.) Starodub. (1, 2); Caltha arctica R. Br. (1, 2); C. palustris L.: a). subsp. membranacea (Turcz.) Hult. (1); б). subsp. palustris (2); Coptidium lapponicum (L.) Tzvelev (1, 2); Delphinium chamissonis G. Pritzel (1, 2); D. ochotense Nevski (1, 2); Pulsatilla angustifolia Turcz. (2); Ranunculus gmelinii DC. (2); R. hyperboreus Rottb. (2); R. monophyllus Ovcz. (2); R. nivalis L. (1, 2); R. pedatifidus Smith. s.l. (2); R. propinquus C. A. Mey. (1, 2); R. pygmaeus Wahlenb. (2); R. turneri Greene: a). subsp. jacuticus (Ovcz.) Tolm. (1, 2); б). subsp. turneri (1); Trollius sibiricus Schipcz. (2).

Fam. Papaveraceae Juss.: Papaver angustifolium Tolm. (1); P. czekanowskii Tolm. (1); P. lapponicum (Tolm.) Nordh. subsp. orientale Tolm. (1, 2); P. leucotrichum Tolm. (1, 2); P. minutiflorum Tolm. (1); P. nudicaule L. subsp. commune Turcz. var. riparia Petrovsky (1); P. paucistaminum Tolm. et Petrovsky (1, 2); P. pulvinatum Tolm.: a). subsp. lenaense Tolm. $(1,2)$; б). subsp. pulvinatum (1).

Fam. Fumariaceae Marquis: Corydalis arctica M. Pop. (1, 2).

Fam. Cruciferae Juss.: Arabidopsis septentrionalis (N. Busch) V. I. Dorof. (1, 2); A. umbrosa (Turcz. ex Steud.) V. I. Dorof. (2); Cardamine bellidifolia L. (1); C. microphylla Adams (1, 2); C. nymanii Gand. (1, 2); C. prorepens Fisch. (1, 2); Cochlearia arctica Schlecht. (1); C. groenlandica L. (1, 2); Descurainia sophioides (Fisch. ex Hook.) O.E. Schulz (1, 2); Dichasianthus humilis (C. A. Mey.) Soják (1, 2); Dimorphostemon pinnatifidus (Willd.) H. L. Yang (1); Draba alpina L. (1, 2); D. cinerea Adams (1, 2); D. fladnizensis Wulf. (1, 2); D. hirta L. (1); D. juvenilis Kom. (1, 2); D. metaarctica Petrovsky (1); D. nivalis Lilyebl. (1); D. pauciflora R. Br. (1, 2); D. pilosa DC. (1); D. subcapitata Simm. (1, 2); Erysimum pallasii (Pursch) Fernald. (1, 2); Eutrema edwardsii R. Br. (1, 2); Parrya nudicaulis (L.) Regel $(1,2)$; Rorippa palustris (L.) Bess. (1); Sphaerotorrhiza trifida (Poir.) A. P. Khokhr. $(1,2)$. 
Fam. Crassulaceae J. St.-Hil.: Rhodiola rosea L. subsp. borealis (Boriss.) Khokhr. et Kuvaev. $(1,2)$.

Fam. Saxifragaceae Juss.: Chrysosplenium alternifolium L. subsp. sibiricum (Ser. ex DC.) Hult. (1, 2); Saxifraga bronchialis L. (1, 2); S. cernua L. (1, 2); S. foliolosa R. Br. (1); S. hieracifolia Waldst. et Kit. (1, 2); S. hirculus L. (1, 2); S. hyperborea R. Br. (2); S. nelsoniana D. Don (1, 2); S. nivalis L.: a). var. nivalis (2); б). var. tenuis Wahlenb. (2); $S$. oppositifolia (2); S. setigera Pursh (1, 2); S. spinulosa Adams (1, 2).

Fam. Parnassiaceae Martinov: Parnassia kotzebuei Cham. et Schlecht. (2); P. palustris L. subsp. neogaea (Fern.) Hulten (2).

Fam. Grossulariaceae DC.: Ribes triste Pall. $(1,2)$.

Fam. Rosaceae Juss.: Acomastylis glacialis (Adams) Khokhr. (1); Comarum palustre L. (1, 2); Dryas punctata Juz. (1, 2); Potentilla hyparctica Malte (1); P. nivea L. s.l. $(1,2)$; P. stipularis L. (1, 2); P. uniflora Ledeb. (1, 2); Rubus chamaemorus L. (1, 2); Sanguisorba officinalis L. var. polygama (Nyl.) Serg. $(1,2)$.

Fam. Fabaceae Lindl.: Astragalus alpinus L. (1, 2); A. frigidus (L.) A. Gray (1, 2); A. norvegicus Grauer (1, 2); A. tugarinovii N. Basil. (2); A. umbellatus Bunge (1, 2); Hedysarum arcticum B. Fedtsch. (1, 2); Oxytropis adamsiana (Trautv.) Jurtz. (1, 2); O. nigrescens (Pall.) Fisch. (1, 2); O. sordida (Willd.) Pers. s.1. (1, 2).

Fam. Empetraceae Hook. et Lindl.: Empetrum nigrum L. s.1. (1, 2).

Fam. Violaceae Batsch: Viola biflora L. $(1,2)$.

Fam. Onaragaceae Juss.: Chamaenerion latifolium (L.) Th. Fries et Lange $(1,2)$; Epilobium davuricum Fisch. ex Hornem. (2); E. palustre L. (1, 2).

Fam. Hippuridaceae Vest.: Hippuris $\times$ lanceolata Retz. (2).

Fam. Umbelliferae Juss.: Angelica decurrens (Ledeb.) B. Fedtsch. (1); Conioselinum tataricum Hoffm. (1); Pachypleurum alpinum Ledeb. (1, 2); Phlojodicarpus villosus (Turcz. ex Fisch. et C. A. Mey.) Ledeb. (1).

Fam. Pyrolaceae Dumort.: Pyrola rotundifolia L. s.1. $(1,2)$.

Fam. Ericaceae Juss.: Arctous alpina (L.) Niedenzu (1, 2); Cassiope tetragona (L.) D. Don $(1,2)$; Ledum palustre L.: a). subsp. decumbens (Ait.) Hult. (1, 2); б). var. angustum N. Busch. $(1,2)$; в). var. dilatatum Wahlenb. $(1,2)$; Rhododendron adamsii Rehder $(1,2)$; Vaccinium uliginosum L.: a). subsp. microphyllum (Lange) Tolm. (1, 2); б). subsp. uliginosum (1); V. vitis-idaea L.: a). subsp. minus (Lodd.) Hult. (1, 2); б). subsp. vitis-idaea $(1,2)$.

Fam. Diapensiaceae Lindl.: Diapensia obovata (Fr. Schmidt) Nakai (1, 2).

Fam. Primulaceae Batsch ex Bork.: Androsace bungeana Schischk. et Bobr. (1, 2); A. septentrionalis L. (1, 2); Primula matthioli (L.) V. A. Richt. subsp. sibirica (Andrz. ex Besser) Kovt. (2).

Fam. Limoniaceae Ser.: Armeria scabra Pall. ex Schult. $(1,2)$.

Fam. Gentianaceae Juss.: Comastoma tenellum (Rottb.) Toyokuni (1, 2).

Fam. Polemoniaceae Juss.: Polemonium acutiflorum Willd. ex Roem. et Schult. $(1,2)$.

Fam. Boraginaceae Juss.: Eritrichium villosum (Ledeb.) Bunge (1, 2); Myosotis asiatica (Vestergen) Schischk. et Serg. (1, 2); M. suaveolens Waldst. et Kit. s.l. (1, 2).

Fam. Lamiaceae Martinov: Thymus extremus Klokov (1, 2); T. reverdattoanus Serg. $(1,2)$.

Fam. Scrophulariaceae Juss.: Castilleja arctica Kryl. et Serg. (2); Lagotis minor (Willd.) Standl. (1, 2); Pedicularis alopecuroides Adams (1); P. amoena Adams ex Stev. (1, 2); P. capitata Adams $(1,2)$; P. lapponica L. $(1,2)$; P. oederi Vahl $(1,2)$; P. sceptrumcarolinum L. (1, 2); P. sudetica Willd. s.1. (1, 2); P. tristis L. (2); P. verticillata L. (1, 2).

Fam. Rubiaceae Juss.: Galium densiflorum Ledeb. (2); G. trifidum L. (1).

Fam. Adoxaceae E. Mey.: Adoxa moschatellina L. (1, 2).

Fam. Valerianaceae Batsch: Valeriana capitata Pall. ex Link. (1, 2). 
Fam. Campanulaceae Juss.: Campanula rotundifolia L. $(1,2)$.

Fam. Asteraceae Bercht. et G. Presl.: Antennaria villifera Boriss. (2); Arnica iljinii (Maguire) Iljin (1, 2); Artemisia arctisibirica Korobkov (1, 2); A. furcata Bieb. (1, 2); A. lagopus Fisch. ex Bess. subsp. abbreviata Krasch. ex Korobkov (1, 2); A. tilesii Ledeb. (1, 2); Chrysanthemum zawadskii (Herb.) Tzvel. subsp. peleiolepis (Trautv.) Zuev (1, 2); Crepis chrysantha (Ledeb.) Turcz. subsp. minor (Ledeb.) Lomonosova (1, 2); C. nana Richards. (1, 2); Packera heterophylla (Fisch.) E. Wiebe (1, 2); Petasites frigidus (L.) Fries $(1,2)$; P. glacialis (Ledeb.) Polun. (1); P. sibiricus (J. F. Gmel.) Dingwall. (2); Saussurea alpina (L.) DC. (1, 2); S. tilesii (Ledeb.) Ledeb. (1, 2); Tanacetum bipinnatum (L.) Sch. Bip. (1, 2); Taraxacum arcticum (Trautv.) Dahlst. (1); T. macilentum Dahlst. (1, 2); T. stepanovae Worosch. (1, 2); Tephroseris integrifolia (L.) Holub (2); Tripleurospermum hookeri Sch. Bip. (1).

The diversity of vascular plants of SF 1 is estimated at 228 species, 11 subspecies and 2 varieties (a total of 241 taxa / km2); in SF 2 - 216 species, 1 notospecies, 11 subspecies and 4 varieties (total 232 taxa $/ \mathrm{km} \mathrm{2}$ ). These are quite high indicators, more typical for the islands of the Lena River in the middle taiga subzone of the Boreal region (Haryyalah Island - 228 taxa / km 2). The quantitative indicators of the standard Chinke and SobolYuryage floras are similar in value, but differ somewhat in species composition. Common to both standard floras are 176 species, 9 subspecies and 2 varieties. The specificity of the standard Chinke flora is formed by 52 species and 2 subspecies (a total of 54 taxa), and in Sobol-Yuryage - 40 species, 1 notospecies, 2 subspecies and 1 variety (a total of 44 taxa).

\section{References}

1. E. Nikolin, BIO Web of Conferences 16 (2019) 